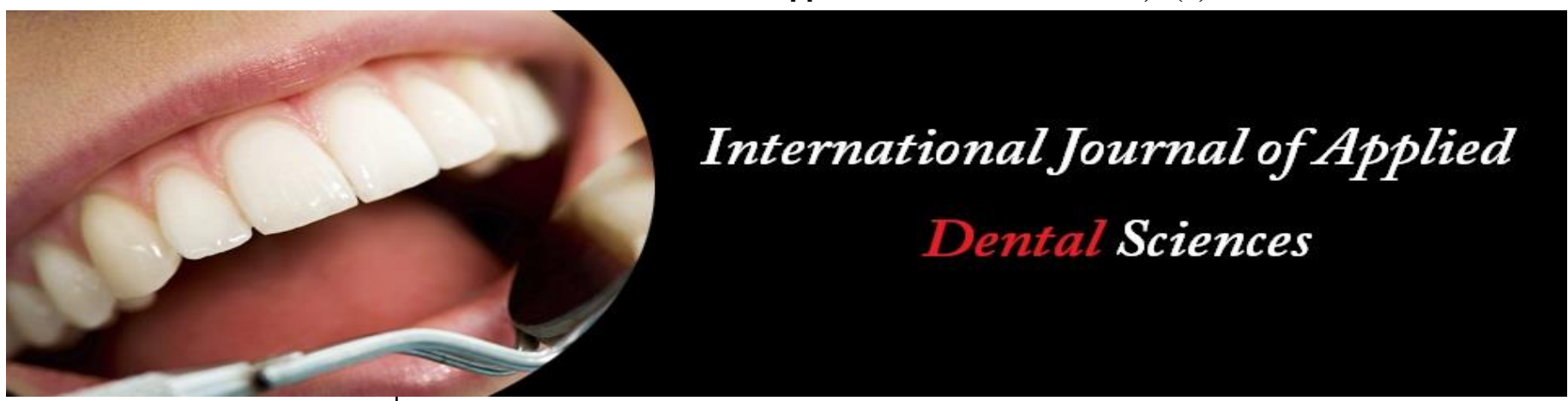

ISSN Print: 2394-7489

ISSN Online: 2394-7497

IJADS 2020; 6(4): 468-473

(C) 2020 IJADS

www.oraljournal.com

Received: 24-08-2020

Accepted: 28-09-2020

Dr. Sheikh Zahid Nazir

Resident, Department of

Orthodontics and Dentofacial

Orthopaedics, Government

Dental College and Hospital,

Srinagar, Jammu and Kashmir, India

Dr. Mohammad Mushtaq Professor and Head of

Department, Department of

Orthodontics and Dentofacial

Orthopaedics, Government

Dental College and Hospital,

Srinagar, Jammu and Kashmir, India
Corresponding Author: Dr. Sheikh Zahid Nazir Resident, Department of Orthodontics and Dentofacial Orthopaedics, Government Dental College and Hospital, Srinagar, Jammu and Kashmir, India

\section{Incisor mandibular plane angle (IMPA), Frankfort mandibular plane angle (FMA) and lower incisor to A-Pog distance, and their relation in different skeletal classes}

\author{
Dr. Sheikh Zahid Nazir and Dr. Mohammad Mushtaq
}

DOI: https://doi.org/10.22271/oral.2020.v6.i4g.1105

\section{Abstract}

Introduction: Tweed's cephalometric analysis has its beginning in clinical orthodontics where he found that the cases of malocclusion with pleasing outcome, harmonious profiles and stable occlusion following orthodontic treatment had a common consistent feature of occlusion: their mandibular incisors were upright on their skeletal bases. So the correct position of incisors is essential for achieving stable end results and establish harmony and balance of facial aesthetics.

Aims and objectives: The purpose of this study was to evaluate the linear and angular position of mandibular incisors over the basal bone and their relationship with frankfort mandibular plane angle in different skeletal classes.

Materials and Methods: A total of 60 lateral cephalograms in the age group of 18-30 years of Class I, Class II, Class III subjects were selected and traced as per inclusion criteria. All the linear and angular measurements were done in each group and their relationship and comparison was done in each group by using ANOVA test and Student's independent t-test.

Results: Descriptive statistical analysis was first carried out to find the means of IMPA, FMA angle and lower incisor to A-pog distance. On doing intergroup comparison with ANOVA test and Student's independent t-test, statistically significant difference was found only in IMPA and lower incisor to A-pog line distance between Class I and III groups and Class II and Class III groups.

Conclusion: There appears to exist a demonstrable relationship between the axial inclination of the mandibular incisors and the incisor mandibular plane angle, the greater the procumbency of the mandibular incisors, the greater the incisor mandibular plane angle in excess of 90 degree and vice versa. There also appears to exist a relationship between the incisor mandibular plane angle and the contour of the lower third of the face. The lower incisors tend to be more upright in subgroups with prognathic mandible than subgroups with normal or retrognathic mandible.

Keywords: Lower incisor, malocclusion, growth pattern

\section{Introduction}

Angle's philosophy of orthodontic treatment was based on the assumption that if the cuspal interdigitation of the teeth was made normal, the stimulation occasioned by orofacial function would result in growth of basal bone structures, i.e maxilla and mandible, which would accommodate full complement of the teeth and result in a balanced and harmonious face. Hence, little or no thought was given to the inclination of mandibular incisors and to the mesiodistal relationship between teeth and their respective jaw bones. However in the years following, Tweed realised his inabilities to create balance and harmony of face in more than a few cases. And noted significant relapse in quite a few of his treated cases thus casting doubt over the long term stability of the results, so Tweed's analysis is essentially based on the inclination of the mandibular incisors to the basal bone and its association with the vertical relation of mandible to the cranium ${ }^{[1-3]}$ His initial impression was based on the clinical examination alone. The relationship of teeth to the basal bone was carefully noted, especially the inclination of incisor teeth. He found in an average non-orthodontic normals, the incisal inclination was approximately $90^{\circ}$ when related to the mandibular plane. From the study, he 
found a variation of $10^{\circ}$ in the inclination of mandibular incisor as related to mandibular plane in subjects with normal occlusion. He concluded that if the orthodontist is to attain facial esthetics and occlusion similar to that found in non orthodontic normals, then the mandibular incisors must be positioned in a range of $85-95^{\circ}$ with a mean of $90^{\circ}$. So the correct position of incisors is essential for achieving stable end results and establish harmony and balance of facial aesthetics.

\section{Aims and objectives}

The main purpose of this study was to evaluate and measure the angular and linear measurements of mandibular incisors by IMPA angle and A-pog linear distance respectively in different skeletal classes and their relation with FMA angle in different skeletal classes and to demonstrate nature's compensation of inclination of mandibular incisors when related to mandibular plane.

\section{Materials and Methods}

A total of 60 lateral cephalometric radiographs of Class I, Class II and Class III subjects were selected by using different parameters like ANB angle ${ }^{[4]}$. Beta angle ${ }^{[5]}$ Wits appraisal ${ }^{[6]}$ and traced as per the inclusion criteria. The patients were selected from the Department of Orthodontics Government Dental College and Hospital Srinagar, Kashmir. Patients in the age group of 18-30 years were selected with complete eruption of all the permanent teeth and all the radiographs were standardised and were taken by the same operator and machine. Patients with craniofacial syndromes like cleft clip and palate and with congenitally missing teeth were excluded from the study. All cephalograms were taken with patients in standing position with teeth in centric occlusion and lips relaxed. All the cephalograms were taken using the same $\mathrm{x}$ ray machine and a standard technique. The machine used was NewTom Giana NNT. No corrections for enlargement were made in the lateral cephalograms as all the cephalograms were taken using the same machine and the same operator. All the films were exposed with $64 \mathrm{KVp}, 8 \mathrm{~mA}$ and an exposure time of 0.9 seconds. All the cephalograms were traced on a standard acetate paper of $8 \times 10$ inch size and 0.003 inch thickness by a standard technique using a soft $3 \mathrm{H}$ pencil using a view box. Tracings were done in a darkened room with no additional light. All the tracings were done by a single observer. Reproducibility was checked by retracing a random $10 \%$ segment of the original sample after a gap of 3 weeks with $0.5 \mathrm{~mm}$ linear ad 0.5 degree angular correction.

\subsection{Parameters defined}

1. Incisor mandibular plane angle (IMPA): The mandibular incisor angle is the inner angle formed between the long axis of mandibular central incisor and the mandibular plane. Its mean value is $90^{\circ}\left(85^{\circ}-95^{\circ}\right)$. A large IMPA suggests proclined mandibular incisors over the basal bone and decreased IMPA suggests retroclined mandibular incisors over the basal bone. ${ }^{[3]}$ (Figure 1)

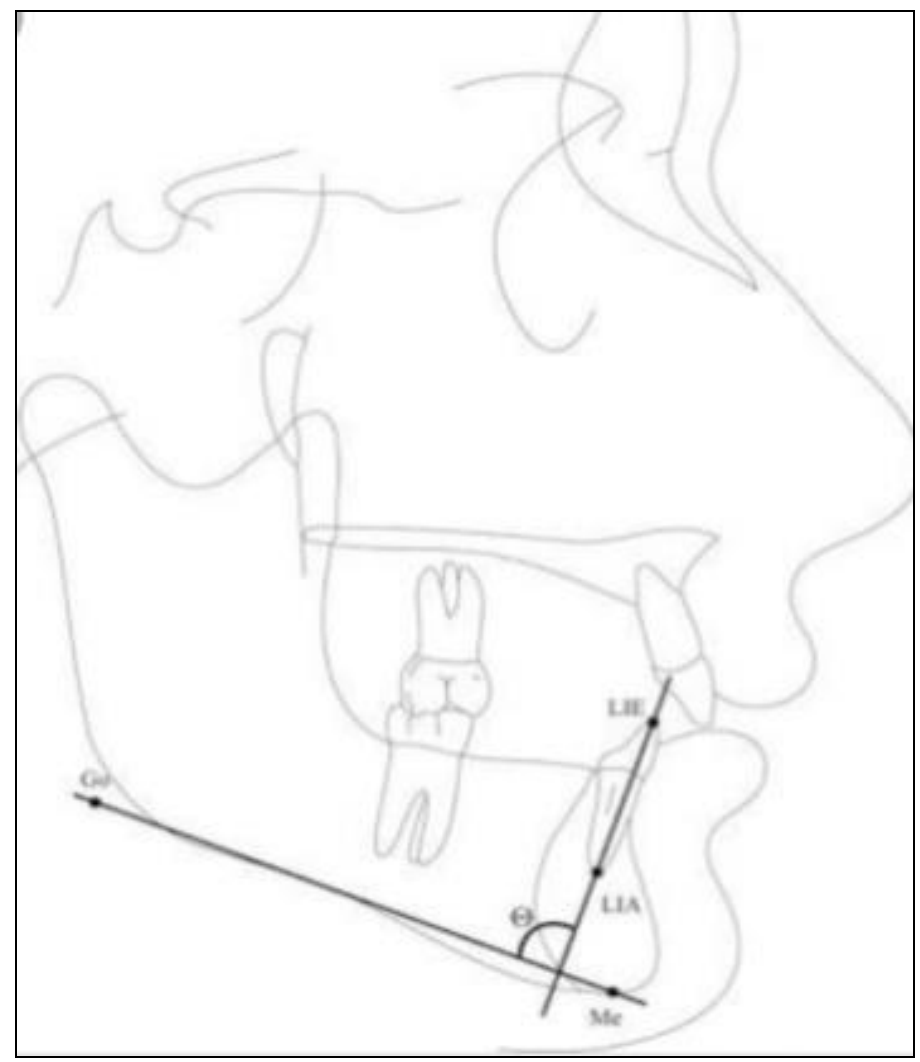

Fig 1: Incisor mandibular plane angle (IMPA)

2. Frankfort mandibular plane angle (FMA): The Frankfort mandibular plane angle is formed by Frankfort plane (Po- Or) and the mandibular plane drawn along the lower border of the mandible. Its mean value is $25^{\circ}$ $\left(16^{\circ}-35^{\circ}\right)^{[3]}$. (Figure 2) 


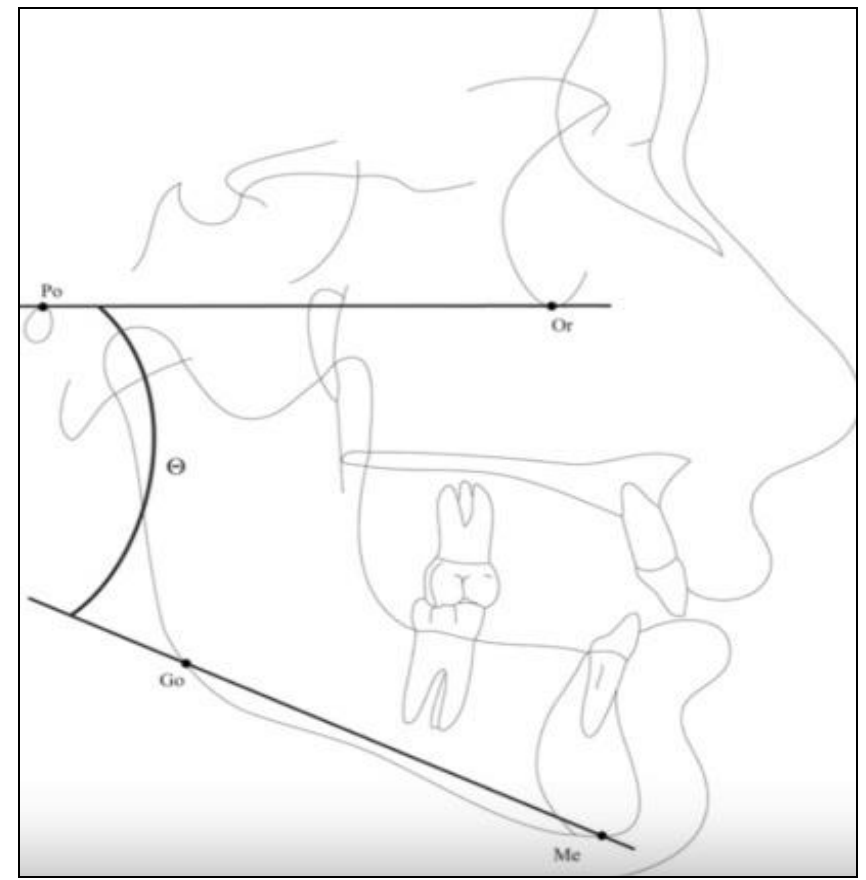

Fig 2: Frankfort mandibular plane angle (FMA)

3. Lower incisor in relation to A-Pog line: The distance is measured between the edge of the mandibular incisor and a line drawn from point $\mathrm{A}$ to pogonion (A-pog line).
In a well-balanced face, the distance should be $1-3 \mathrm{~mm}$ ${ }^{[10]}$. (Figure 3)

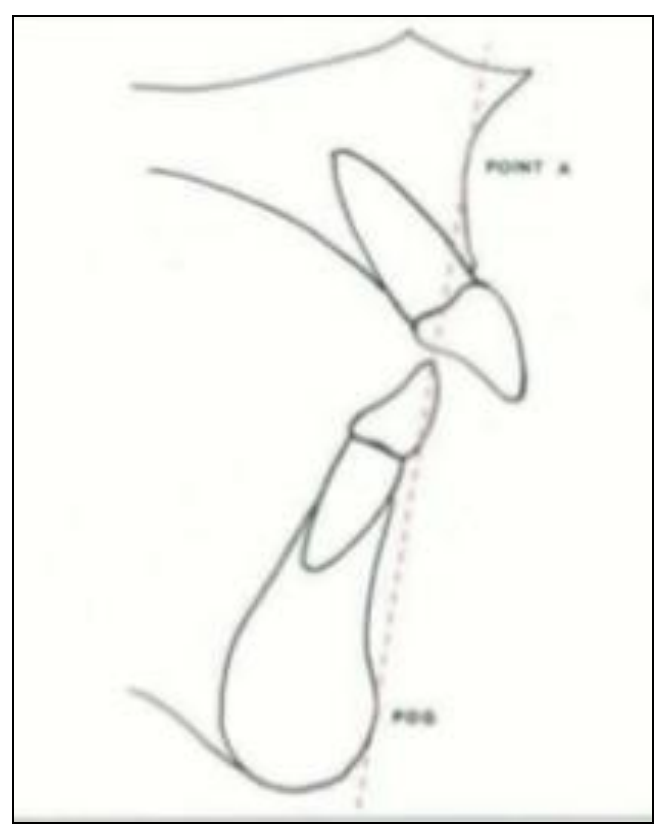

Fig 3: Lower incisor in relation to A-Pog line

\subsection{Stastistical analysis}

The recorded data was compiled and entered in a spreadsheet (Microsoft Excel) and then exported to data editor of SPSS Version 20.0 (SPSS Inc., Chicago, Illinois, USA). Statistical software SPSS (version 20.0) and Microsoft Excel were used to carry out the statistical analysis of data. Data were expressed as Mean \pm SD. Analysis of variance (ANOVA) and Student's independent t-test were employed for inter group analysis of data. Graphically the data was presented by bar diagrams. A P-value of less than 0.05 was considered statistically significant. All P-values were two tailed as shown in tables $(1,2)$ below.

Table 1: Showing descriptive statistics of cephalometric variables according to malocclusion groups

\begin{tabular}{|c|c|c|c|c|c|c|}
\hline \multirow{2}{*}{ Cephalometric variable } & \multicolumn{2}{|c|}{ Class I } & \multicolumn{2}{|c|}{ Class II } & \multicolumn{2}{c|}{ Class III } \\
\cline { 2 - 7 } & Mean & SD & Mean & SD & Mean & SD \\
\hline Lower incisor to mandibular plane angle (IMPA Angle) & 91.35 & 6.49 & 93.75 & 6.62 & 81.60 & 10.05 \\
\hline Lower incisor to A Pog Line & 2.10 & 1.33 & 1.55 & 2.33 & 3.50 & 2.09 \\
\hline FH mandibular plane angle & 27.60 & 6.44 & 27.30 & 6.22 & 27.10 & 8.32 \\
\hline
\end{tabular}


Table 2: Intergroup based on cephalometric variables among various malocclusion groups

\begin{tabular}{|c|c|c|c|}
\hline \multirow{2}{*}{ Cephalometric variable } & \multicolumn{3}{|c|}{ P-value } \\
\cline { 2 - 4 } & I vs II & I vs III & II vs III \\
\hline Lower incisor to mandibular plane angle (IMPA Angle) & 0.341 & $<0.001^{*}$ & $<0.001^{*}$ \\
\hline Lower incisor to A Pog Line & 0.379 & $0.028^{*}$ & $0.003^{*}$ \\
\hline FH to mandibular plane angle & 0.893 & 0.823 & 0.929 \\
\hline
\end{tabular}

\subsection{Lower incisor to mandibular plane angle (IMPA} Angle)

In Class I subjects the mean of Lower incisor to mandibular plane angle (IMPA Angle) in lateral cephalogram is 91.35 with a standard deviation of 6.49. In Class II subjects the mean of Lower incisor to mandibular plane angle (IMPA Angle) in lateral cephalogram is 93.75 with a standard deviation of 6.62. In Class III subjects the mean of Lower incisor to mandibular plane angle (IMPA Angle) in lateral cephalogram is 81.60 with a standard deviation of 10.05 . On intergroup comparison of Lower incisor to mandibular plane (IMPA) in lateral cephalogram, the P-value of comparison between Class I and II is 0.341 which is statistically insignificant. The P-value of intergroup comparison between Class I and III is less than 0.001 which is statistically significant whereas between Class II and III is less than 0.001 which is statistically significant (Tables 1,2 and Fig 1,2).

\subsection{Lower incisor to A-Pog line distance}

In Class I subjects the mean of Lower incisor to A Pog line in lateral cephalogram is 2.10 with a standard deviation of 1.33 . In Class II subjects the mean of Lower incisor to A Pog line in lateral cephalogram is 1.55 with a standard deviation of 2.33. In Class III subjects the mean of Lower incisor to A Pog line in lateral cephalogram is 3.50 with a standard deviation of 2.09. On intergroup comparison of lower incisor to A Pog line the P-value of comparison between Class I and II is 0.379 which is statistically insignificant. The P-value of intergroup comparison between Class I and III is 0.028 which is statistically significant whereas between Class II and III is 0.003 which is statistically significant (Tables 1,2 and Fig $1,2)$..

\subsection{Frankfort mandibular plane angle (FMA angle)}

In Class I subjects the mean of FH mandibular plane angle in lateral cephalogram is 27.60 with a standard deviation of 6.44 . In Class II subjects the mean of FH mandibular plane angle in lateral cephalogram is 27.30 with a standard deviation of 6.22 . In Class III subjects the mean of FH mandibular plane angle in lateral cephalogram is 27.10 with a standard deviation of 8.32. On intergroup comparison of $\mathrm{FH}$ mandibular plane angle the P-value of comparison between Class I and II is 0.893 which is statistically insignificant. The P-value of intergroup comparison between Class I and III is 0.823 which is statistically insignificant whereas between Class II and III is 0.929 which is statistically insignificant (Tables 1,2 and Fig $1,2)$.

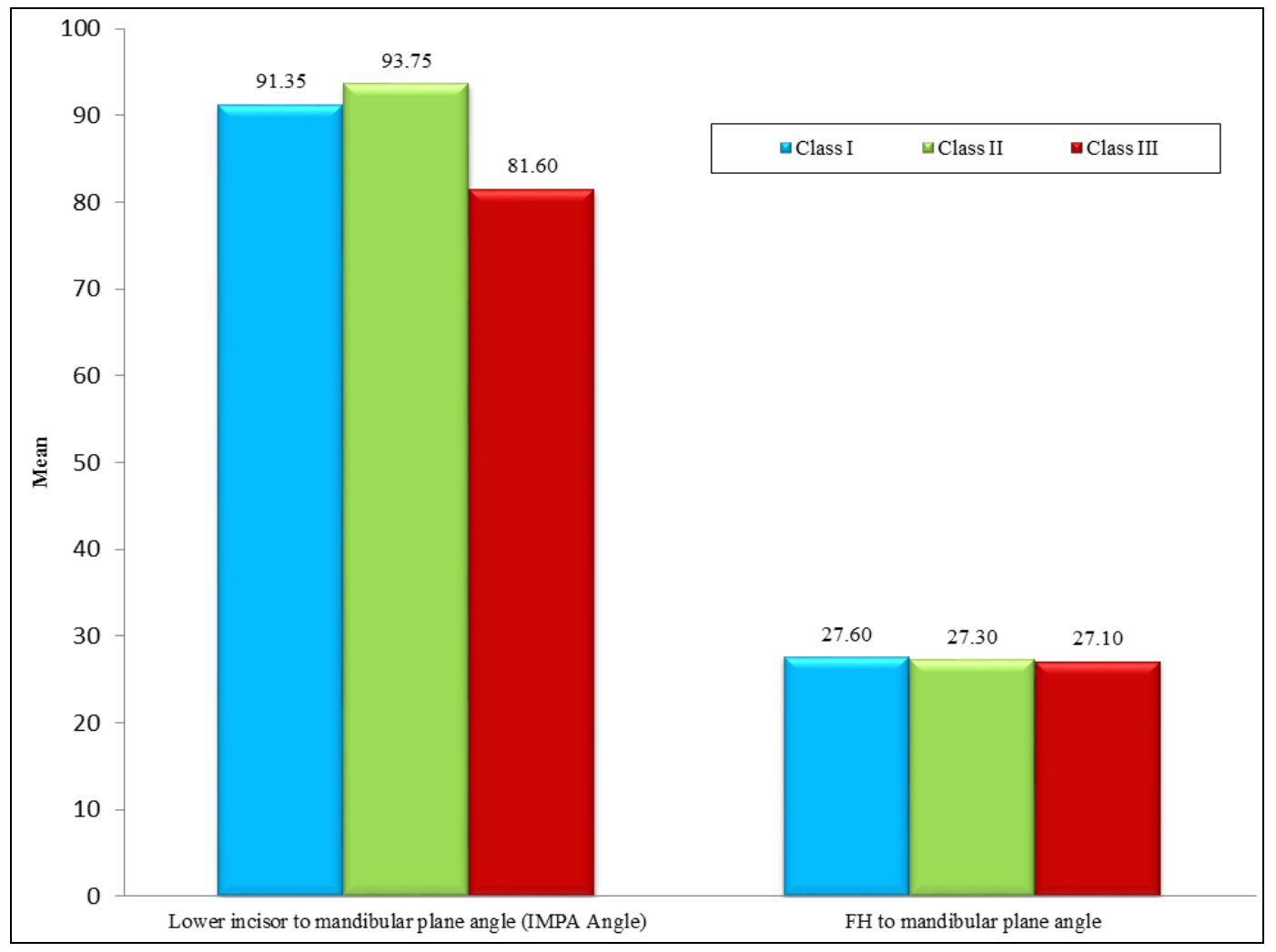

Fig 1: IMPA and FH to mandibular plane angle in various malocclusion subgroups 


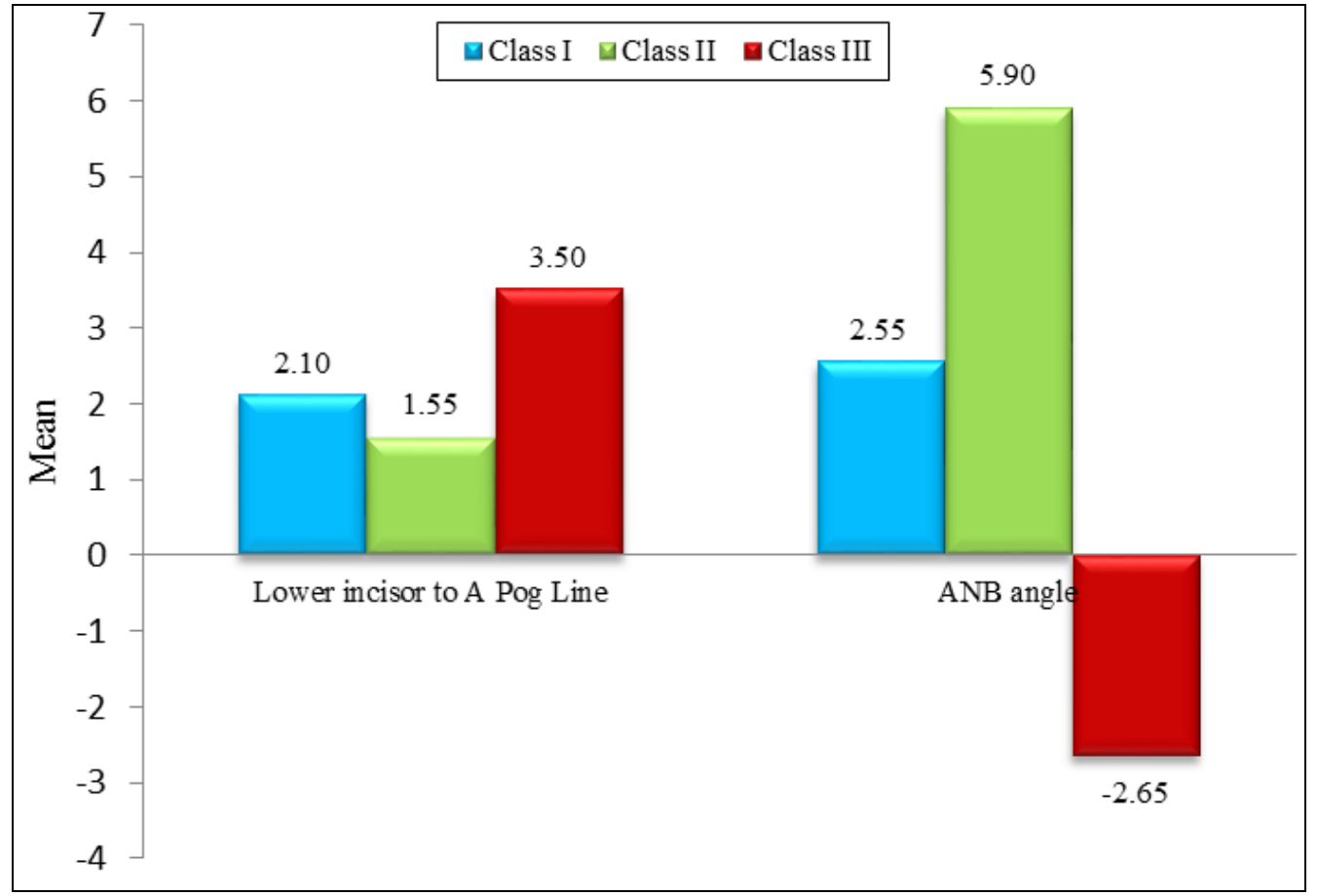

Fig 2: lower incisor to A Pog line in different malocclusion subgroups

\section{Discussion}

Cephalometric radiography is a valuable tool in orthodontic diagnosis and treatment planning and cephalometric values affect decision to treat extraction or non-extraction. Angle's philosophy of orthodontic treatment was based on the assumption that if the cuspal interdigitation of the teeth was made normal, the stimulation occasioned by orofacial function would result in growth of basal bone structures, i.e; maxilla and mandible, which would accommodate full complement of the teeth and result in a balanced and harmonious face. Hence, little or no thought was given to the inclination of mandibular incisors and to the mesiodistal relationship between teeth and their respective jaw bones. However in the years following, Tweed realised his inabilities to create balance and harmony of face in more than a few cases. And noted significant relapse in quite a few of his treated cases thus casting doubt over the long term stability of the results, so Tweed's analysis is essentially based on the inclination of the mandibular incisors to the basal bone and its association with the vertical relation of mandible to the cranium ${ }^{[1-3]}$. He found in an average non-orthodontic normals, the incisal inclination was approximately $90^{\circ}$ when related to the mandibular plane. From the study, he found a variation of $10^{\circ}$ in the inclination of mandibular incisor as related to mandibular plane in subjects with normal occlusion. He concluded that if the orthodontist is to attain facial esthetics and occlusion similar to that found in nonorthodontic normals, then the mandibular incisors must be positioned in a range of $85-95^{\circ}$ with a mean of $90^{\circ}$. Based on his observations on lower incisor mandibular plane angle (IMPA) and its association with variations in the frankfort mandibular plane angle (FMA), he found that a consistent finding was a resultant $3^{\text {rd }}$ angle which was frankfort mandibular incisor angle (FMIA). Tweed found that extraction of teeth was necessary in patients with FMA more than $30^{\circ}$. He observed it was physically impossible to fully compensate the inclination of the mandibular incisors i.e. make them less than upright when FMA ranges upwards from $35^{\circ}$. Prognosis in not good in such cases and the orthodontist is limited in his efforts to create stable end results and establish balance and harmony of facial aesthetics. There appears to exist a demonstrable relationship between the axial inclination of the mandibular incisors and the incisor mandibular plane angle, the greater the procumbency of the mandibular incisors, the greater the incisor mandibular plane angle in excess of 90 degree and vice versa. There also appears to exist a relationship between the incisor mandibular plane angle and the contour of the lower third of the face. ${ }^{[7]}$ In this study the mean values of incisor mandibular plane angle was $91.35^{\circ}$ in Class I subjects, $93.75^{\circ}$ in Class II subjects and $81.60^{\circ}$ in Class III subjects. These values suggest a tendency of lower incisors to be more upright in subgroups with prognathic mandible than subgroups with normal or retrognathic mandible. Tweed ${ }^{[1]}$ established the importance of the relation between the inclination of the inferior incisor and the mandibular plane, establishing between them a determined angular measure. In our study, we found similar results. It was observed statistically significant relation was found between the inclination of the lower incisor and the mandibular plane in the different malocclusions. Intergroup comparison of lower incisor mandibular plane angle between Class I and Class II subgroups was statistically insignificant where as intergroup comparison between Class II and Class III and between Class I and Class III was statistically significant. Class II and Class III subjects represent two extremes of range of spatial position of mandible and as such significant difference of IMPA between these two groups is expected. The fact that comparison between Class I and Class III subgroups is statistically significant where as comparison between Class I and Class II subgroups is not, suggests that incisors are more upright in subgroups with prognathic mandible than they are procumbent in subgroups with retrognathic mandible. During growth there occurs an overall change in the orientation of each jaws judged by the palatal plane and mandibular plane, it results from a combination of internal and external rotation. It is easier to visualize the internal and external rotation of the jaws by considering the mandible first and the final direction of growth pattern is the determined by the combination of internal and external rotation of the mandible ${ }^{[8]}$ which can be measured by various 
cephalometric parameters. Frankfort mandibular plane angle (FMA) is one of the most important measurements required for orthodontics, dentofacial orthopedics and orthognathic surgical treatment planning. FMA is commonly used to indicate the vertical or horizontal growth pattern of patients. The current study was carried out to evaluate the measurements of FMA in lateral cephalogram and correlation of this measurement among various sub groups of malocclusion. The mean value of FMA angle in Class I subgroup was 27.60, 27.30 in Class II and 27.10 in Class III. On studying intergroup comparison of FMA among various sub groups of malocclusion no statistically significant difference was found among different groups. Tweed [1] reports in his article how Downs stated using, at the beginning, the plane A-Pog to evaluate the position of the lower incisor, and the modifications that it has to be done during the orthodontic treatment. In our study the mean values of lower incisor to A-Pog line was 2.10 in Class I subjects, 1.55 in Class II subjects and 3.50 in Class III subjects. Similar to findings of incisor mandibular plane angle the values of lower incisor to A-Pog line suggest a tendency of lower incisors to be more upright in subgroups with prognathic mandible than subgroups with normal or retrognathic mandible ${ }^{[9]}$. Intergroup comparison of lower incisor to A-Pog line between Class I and Class II subgroups was statistically insignificant where as intergroup comparison between Class II and Class III and between Class I and Class III was statistically significant. As stated above Class II and Class III subjects represent two extremes of range of spatial position of mandible and as such significant difference of IMPA between these two groups is expected. The fact that comparison between Class I and Class III subgroups is statistically significant where as comparison between Class I and Class II subgroups is not, suggests that incisors are more upright in subgroups with prognathic mandible than they are procumbent in subgroups with retrognathic mandible.

\section{Conclusion}

There appears to exist a demonstrable relationship between the axial inclination of the mandibular incisors and the incisor mandibular plane angle, the greater the procumbency of the mandibular incisors, the greater the incisor mandibular plane angle in excess of 90 degrees and vice versa. There also appears to exist a relationship between the incisor mandibular plane angle and the contour of the lower third of the face. The lower incisors tend to be more upright in subgroups with prognathic mandible than subgroups with normal or retrognathic mandible. Lower incisor to A-Pog line suggest a tendency of lower incisors to be more upright in subgroups with prognathic mandible than subgroups with normal or retrognathic mandible. So the space requirements in the arch should be calculated based on the amount of change required to place the lower incisors correctly over the basal bone which is termed as head plate correction.

\section{References}

1. Tweed $\mathrm{CH}$. The Frankfort-mandibular incisor angle (FMIA) in orthodontic diagnosis, treatment planning and prognosis. Angle Orthod 1954;24:121-169.

2. Tweed $\mathrm{CH}$. Was the development of the diagnostic facial triangle an accurate analysis based on fact or fancy? Am J Orthod 1962;48:823-40.

3. Tweed $\mathrm{CH}$. The diagnostic facial triangle in the control of treatment. Am J Orthod 1969;55(6):651-7.
4. Steiner CC. Cephalometrics for you and me. Am J Orthod 1953;39:729-55.

5. Baik CY, Ververeidou M. A new approach of assessing sagittal discrepancies: The Beta angle. Am J of Orthod and Dentofacial Orthop 2004;126(1):100-105.

6. Roth R. The Wits appraisal- its skeletal and dentoalveolar background. Eur J Orthod 1982;4:21-8.

7. Fish SF. Change in the gonial angle. J oral Rehabil 1979;6:219-227.

8. Larheim TA, Svanaes DB. Reproducibility of rotational panoramic radiography: mandibular linear dimensions and angles. Am J Orthod Dentofacial Orthop 1986;90:4551.

9. Houston WJB, Edler R. Long-term stability of the lower labial segment relative to the A-Pog line. EJO 1990;12(3):302-310.

10. McNamara JA. A method of cephalometric evaluation. Am J Orthod 1984;86(6):449-69. 